\title{
Rotura uterina en gestante de 13 semanas. Embarazo ectópico intersticial
}

\author{
María Gador Manrique $F^{1}$, Rosario Redondo A. ${ }^{1}$, Luís Delgado M. ${ }^{1}$, Valois González A. ${ }^{1}$ \\ Leonardo Mauro Aisa ${ }^{1}$, Longinos Aceituno $\mathrm{V}^{1}$
}

Servicio de Obstetricia y Ginecología, Hospital La Inmaculada, Huércal-Overa. Área de Gestión Sanitaria Norte de Almería, Servicio Andaluz de Salud. Almería, Andalucía. España.

\section{RESUMEN}

El embarazo ectópico intersticial es una entidad rara pero con una elevada tasa de mortalidad. El diagnóstico puede resultar difícil y tardío, dada la localización del embarazo en una porción intrauterina de la trompa de Falopio. La gestación puede evolucionar de manera asintomática hasta el segundo trimestre de la gestación, y debutar con una rotura uterina y shock hipovolémico por la proximidad del saco gestacional a la arteria uterina. El tratamiento suele consistir en una resección cornual por vía laparoscópica, aunque se individualizará en función de cada caso, primando ante todo la clínica de la paciente. Se expone el caso de un embarazo ectópico intersticial que debutó con rotura uterina a las 13 semanas de gestación.

\section{PALABRAS CLAVE: Embarazo ectópico intersticial, rotura uterina, mortalidad materna}

\section{SUMMARY}

Interstitial ectopic pregnancy is a rare but with a high mortality rate entity. Diagnosis can be difficult and late, given the location of the pregnancy in an intrauterine portion of the fallopian tube. Pregnancy can evolve asymptomatic until the second trimester, and debuting with uterine rupture and hypovolemic shock due to the proximity of the gestational sac to the uterine artery. Treatment usually consists of a laparoscopic cornual resection, although it will be individualized according to each case, giving priority to the patient clinic condition. The case of an interstitial ectopic pregnancy who presented with uterine rupture at 13 weeks of gestation is presented.

\section{KEY WORDS: Interstitial ectopic pregnancy, uterine rupture, maternal mortality}

\section{INTRODUCCIÓN}

Pese al incremento de la incidencia de las gestaciones ectópicas en los últimos años, debido principalmente al aumento en las técnicas de reproducción asistida, mayor frecuencia de enfermedad pélvica inflamatoria y mayores antecedentes de cirugía pélvica, el avance en métodos diagnósticos (ecografía, determinación de beta hCG) ha permitido que el diagnóstico pueda realizarse de manera precoz, pudiéndose realizar un tratamiento más conservador y disminuyendo las tasas de mortalidad.

No ocurre así en el caso del embarazo ectópico intersticial, ya que al localizarse en una porción de la trompa de Falopio alojada en el espesor del miometrio, con una gran vascularización, puede, por un lado, crecer de manera asintomática hasta más avanzada la gestación que en las demás localizaciones de embarazos ectópicos, y por otro, causar 
una hemorragia masiva por la detección del cuadro tras la rotura uterina en ese nivel (1).

El embarazo intersticial supone del 2 al $4 \%$ de todos los embarazos ectópicos, y presenta una alta tasa de mortalidad materna, del 2 al 2,5 \% (dos veces mayor que las demás localizaciones), lo cual está en relación con la frecuente detección tardía del cuadro dada la dificultad que en ocasiones supone el diagnóstico (2).

El objetivo de esta comunicación es presentar un caso clínico de un embarazo ectópico intersticial que debutó con un cuadro de abdomen agudo y shock hipovolémico, en una paciente sin factores de riesgo a las 13 semanas de gestación.

\section{Caso clínico}

Paciente de 32 años, cursando una gestación de 13 semanas. Entre sus antecedentes ginecoobstétricos destaca un aborto espontáneo a las 8 semanas de gestación dos años antes, que no requirió de legrado. Menarquia a los 16 años. Con antecedentes personales de obesidad y sin antecedentes quirúrgicos.

Gestación conseguida de manera espontanea. La primera visita obstétrica se realizó una semana antes (12 semanas) encontrándose la paciente asintomática, en la ecografía se visualiza saco gestacional intrauterino con embrión con actividad cardíaca (AC) positiva, longitud cráneo-caudal (CRL) de $61 \mathrm{~mm}$ (acorde a 12 semanas), sin malformaciones aparentes, translucencia nucal (TN): $2,0 \mathrm{~mm}$. Beta hCG y PAPP-A dentro del rango de la normalidad. Riesgo combinado de Síndrome de Down: 1/91 (pendiente de informar a la paciente y ofrecerle prueba diagnóstica prenatal para estudio cromosómico).

Acude a urgencias por dolor abdominal muy intenso generalizado de tipo cólico, que va en aumento, desde hace unas horas, localizado principalmente en hipogastrio, acompañado de vómitos. Al examen se encuentra en regular estado general. Consciente, orientada, pálida, sudorosa. PA: 80/60, FC: $115 \mathrm{x}$ '. Abdomen distendido, muy doloroso en reposo y a la palpación superficial y profunda de manera difusa, Blumberg dudoso. Sin metrorragia. Al examen pélvico el cuello uterino estaba cerrado, y doloroso a la movilización.

La ecografía abdominal y transvaginal fue muy dificultosa por dolor y obesidad. Se aprecia feto con CRL acorde a semana 13 SG con actividad cardiaca presente, de difícil visualización por anhidramnios. Abundante líquido libre abdominal y material ecógenico mal definido alrededor de útero.

El hemograma reveló una hemoglobina de 10,9 $\mathrm{g} / \mathrm{dL}$, hematocrito de $34 \%$, leucocitos 23.100 (84\% neutrófilos). Las pruebas de coagulación fueron normales.

Ante el diagnóstico de abdomen agudo en gestante de 13 semanas, se indica y realiza intervención urgente. Inmediatamente a la entrada en quirófano la paciente presenta shock hipovolémico, con hemoblogina de $4,9 \mathrm{~g} / \mathrm{dL}$, hematocrito de $15 \%$.

La intervención se inicia mediante laparoscopia, pero dada la inestabilidad hemodinámica de la paciente y el abundante hemoperitoneo que dificulta la correcta visualización de la cavidad pélvica, se decide conversión a laparotomía previéndose una intervención más rápida. Tras la apertura de la cavidad abdominal se extraen gran cantidad de coágulos y sangre, cuantificándose en 2,5 litros de hemoperitoneo. Se visualiza feto y placenta en cavidad abdominal y rotura de útero de unos $4 \mathrm{~cm}$ en tercio lateral derecho de fondo uterino (cuerno uterino derecho) con acceso a cavidad endometrial (Figuras 1 y 2). Anejos normales. Se realiza extracción del feto y placenta de la cavidad abdominal (Figura 3), resección de zona afecta y limpieza de restos deciduocoriales con legrado a través de la rotura uterina. La hemostasia se consigue con electrocoagulación y puntos hemostáticos. Se realiza histerorrafia de miometrio con tres capas de puntos sueltos dobles y sutura continua en serosa uterina. Se respetan ambas trompas ya que no muestran compromiso. Durante la intervención se transfunden 4 concentrados de hematíes y uno de plasma fresco.

El posoperatorio cursa sin incidencias, se administran otros 4 concentrados de hematíes y uno de plasma fresco por anemia aguda. Es dada de alta, tras buena evolución, al tercer día postoperatorio.

La anatomía patológica informa feto de sexo masculino de 13 semanas de gestación, biometrías corporales acordes a edad gestacional. Edad tisular acorte a edad gestacional. Congestión vascular generalizada. Placenta y membranas con presencia de amplias áreas hemáticas y autolisis intensa. Cordón sin alteraciones relevantes.

En el control poscirugía, la paciente se encuentra asintomática, la exploración es normal y la ecografía transvaginal muestra útero y anexos sin alteraciones.

\section{DISCUSIÓN}

Un embarazo ectópico intersticial puede progresar sin sintomatología hasta su debut con una rotura uterina, habiéndose descrito casos diagnosticados incluso en la semana 16. Está descrito que hasta el $50 \%$ de los embarazos ectópicos intersticiales se manifiestan con una rotura uterina (3).

El embarazo crece en una porción de la trompa intrauterina, en la que existe una gran cantidad de miometrio que lo rodea, complaciente y con una gran vascularización. Ello permite que se dilate de forma indolora y que el embarazo pueda progresar hasta una edad gestacional más avanzada que el resto de los embarazos ectópicos localizados en otras porciones de la trompa de Falopio. 


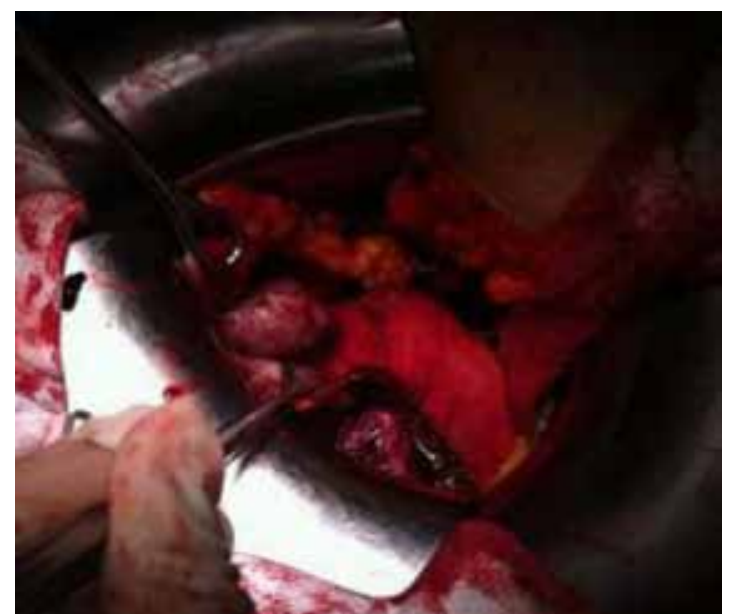

Figura 1. Rotura uterina en tercio lateral derecho de fondo uterino.

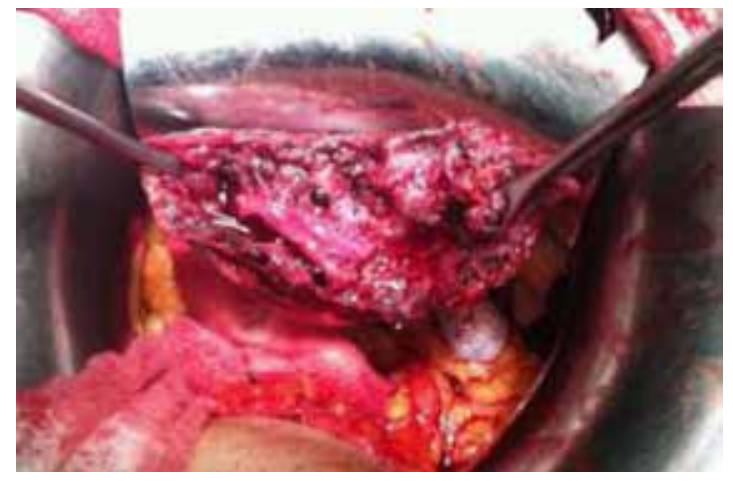

Figura 2. Rotura uterina con acceso a cavidad endometrial.

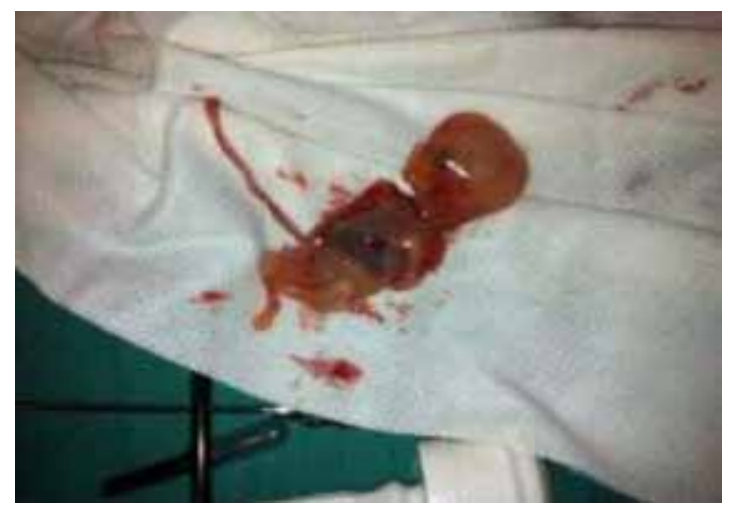

Figura 3. Feto de 13 semanas extraído desde cavidad abdominal.
Por otro lado, la porción intramural de la trompa está muy cercana a la arteria uterina, motivo por el que la rotura uterina a dicho nivel puede resultar en una hemorragia incontrolable, representando una elevada morbilidad y alta tasa de mortalidad, cifrándose hasta en un $2,5 \%$ de los casos.

Los factores de riesgo del embarazo ectópico cornual no difieren de los del resto de embarazos ectópicos (antecedente de enfermedad pélvica inflamatoria, cirugía pélvica previa, malformación uterina, salpingectomía unilateral, portadora de dispositivo intrauterino, técnicas de reproducción asistida, etc.), y la clínica engloba un amplio espectro de manifestaciones: desde cuadros asintomáticos hasta shock hipovolémico, abarcando todos los síntomas que se presentan en los demás embarazos tubáricos (amenorrea, dolor abdominal, metrorragia, etc.). Analíticamente, la hormona beta-hCG puede presentar valores acordes a la edad gestacional (4).

La ecografía tiene un papel fundamental en el diagnóstico, aunque ésta suele resultar difícil y en muchas ocasiones se puede diagnosticar erróneamente una gestación de localización intrauterina normal, ya que el saco gestacional se encuentra, aunque excéntrico, a nivel intrauterino.

Existen tres criterios ecográficos para el diagnóstico de embarazo ectópico intersticial: 1. Cavidad endometrial vacía. 2. Saco gestacional excéntrico en relación a cavidad endometrial. 3. Lecho miometrial delgado. Pese a la presencia de estos tres criterios, se plantea el diagnóstico diferencial con útero septado, útero didelfo y útero bicorne (5). El diagnóstico, por tanto, no es fácil, habiéndose descrito incluso falsos negativos tras la realización de una laparoscopia diagnóstica.

En nuestro caso, la paciente no manifestó ningún tipo de síntoma hasta la semana 13, los valores de beta hCG correspondían a la edad gestacional, y no presentaba ningún factor de riesgo para embarazo ectópico. Durante la primera visita obstétrica no había ninguna sospecha de embarazo ectópico y la ecografía realizada no resultó diagnóstica. Todo ello propició que el diagnóstico se retrasara y no se produjese hasta el debut en forma de abdomen agudo y rápida evolución a shock hipovolémico.

La ecografía realizada en urgencias ante el cuadro clínico de la paciente, tampoco resultó diagnóstica, dada la dificultad técnica por el dolor de la paciente y la mala transmisión ultrasónica por la obesidad, la gran cantidad de hemoperitoneo en forma de coágulos y el anhidramnios. Tanto el hemoperitoneo como el anhidramnios eran consecuencia de la rotura uterina que se estaba produciendo inmediantamente al ingreso, no antes, ya que el producto de la gestación aún estaba vivo, y la inestabilidad hemodinámica no llegó a reflejarse en la analítica realizada al ingreso. 
Es importante resaltar la importancia de la clínica, que prima sobre la analítica. En este caso, la clínica que presentaba la paciente, pese a una analítica no comprometida, fue lo que propició la rápida actuación que permitió que el shock hipovolémico se produjese con la paciente ya en quirófano, evitándose un posible fatal desenlace.

Como en los demás tipos de embarazos ectópicos, es posible el tratamiento médico o el quirúrgico, individualizándose cada caso. Aproximadamente, el $35 \%$ de las mujeres con embarazo ectópico son susceptibles de tratamiento médico, de las cuales el tratamiento será exitoso en casi el $90 \%$, siempre que las pacientes sean seleccionadas adecuadamente (6). El manejo expectante también es posible en casos muy seleccionados de embarazos intersticiales no viables y con valores decrecientes de beta-hCG (7).

Está indicado el tratamiento médico con methotrexate cuando el diagnóstico es temprano, siendo el tratamiento de elección en pacientes asintomáticas que desean conservar la fertilidad. Son candidatas a tratamiento médico aquellas pacientes que cumplan los siguientes criterios (8): estabilidad hemodinámica, posibilidad de seguimiento postratamiento, concentración de beta-hCG $<5000 \mathrm{mU} / \mathrm{mL}$, embarazo ectópico $<3-4 \mathrm{~cm}$ y no presencia de actividad cardíaca. Los siguientes criterios son contraindicaciones absolutas para instaurar tratamiento médico con methotrexate (9): inestabilidad hemodinámica, signos de ruptura del embarazo ectópico, anormalidad hematológica (renal o hepática), inmunodeficiencia, hipersensibilidad al metotrexate, coexistencia con gestación intrauterina viable (gestación heterotópica), imposibilidad de control posterior a la administración del metotrexate.

En el caso de los embarazos ectópicos intersticiales, ni los valores de beta-hCG ni el diámetro del saco gestacional son buenos predictores de respuesta al tratamiento, ya que la rotura de estos embarazos ectópicos depende de la proximidad a la pared uterina.

El tratamiento quirúrgico suele consistir en una resección cornual o, en algunos casos en los que no se puede controlar la hemorragia, una histerectomía (10). Siempre que sea posible está indicada la realización de la intervención menos agresiva con vistas a un futuro embarazo. En algunos casos de gestaciones cornuales pequeñas (inferiores a $4 \mathrm{~cm}$ ) se podría plantear la cuernostomía, evitándose así la resección cornual, ya que la probabilidad de rotura uterina en gestaciones posteriores es significativamente menor (11). También están descritos casos en la literatura de embarazos ectópicos intersticiales tratados con embolización de las arterias uterinas (12). La vía de acceso de primera elección es la laparoscópica, aunque si la paciente presenta inestabilidad hemodinámica está justificada la realización de una laparotomía si se prevé una más rápida hemostasia y así resolución del cuadro (13).

En el caso que nos ocupa, el tratamiento médico no era una opción, ya que el diagnóstico no fue temprano. La vía de acceso fue la laparoscopia dada la estabilidad hemodinámica en el momento de ingreso en quirófano, aunque tuvo que ser reconvertida a laparotomía por el shoch hipovolémico que presentó la paciente inmediatamente tras el comienzo de la intervención, ya que el acceso abierto nos pareció mejor opción para una más rápida actuación ante el hallazgo laparoscópico de un hemoperitoneo abundante. La técnica quirúrgica no precisó cirugía radical al conseguir cohibir la hemorragia con puntos hemostáticos. Fue suficiente con la resección de la porción cornual donde se ubicaba el embarazo, limpieza de restos abortivos mediante legra grande y posterior histerorrafia con varias capas de puntos. El postoperatorio cursó sin incidencias, administrándose un total de 8 concentrados de hematíes y 2 de plasma fresco. La paciente se encontraba asintomática en la revisión posquirúrgica realizada un mes después.

En relación al pronóstico reproductivo de la paciente, la literatura expone una incidencia de recidiva de embarazo ectópico del $15 \%$, no existiendo diferencias tras la realización de tratamiento médico o quirúrgico (14). No hay estudios concluyentes sobre la posibilidad de embarazo tras la resección cornual por embarazo ectópico intesticial, aunque sí se recomienda la realización de una cesárea electiva en caso de gestación posterior (15).

\section{CONCLUSIÓN}

La presentación del embarazo ectópico intersticial es una entidad muy poco frecuente, pero con una alta tasa de mortalidad materna. Por su ubicación intrauterina en una zona muy vascularizada y complaciente, el embarazo pueda progresar hasta entrada la gestación en el segundo trimestre. Ello, unido a la dificultad diagnóstica por poder confundirse ecográficamente el ectópico con un embarazo intrauterino, supone una demora diagnóstica y una mayor tasa de mortalidad en relación a los demás tipos de gestaciones ectópicas. Dicha tasa no está disminuyendo como ocurre con el resto. El manejo convencional es la resección cornual o histerectomía, aunque en casos asintomáticos se puede optar por el tratamiento médico. La vía de acceso de elección es la laparoscopia, aunque la laparotomía queda justificada en una paciente con inestabilidad hemodinámica. El tratamiento oportuno dependerá de la clínica, analítica, experiencia del cirujano y deseo genésico de la paciente. 


\section{REFERENCIAS}

1. Anderson FW, Hogan JG, Ansbacher R. Sudden death: ectopic pregnancy mortality. Obstet Gynecol 2004;103:1218-23.

2. Tulandy T. Clinical manifestations, diagnosis, and management of ectopic pregnancy. UpToDate, 2014.

3. Chan LY, Fok WY, Yuen PM. Pitfall in diagnosis of interstitial pregnancy. Acta Obstet Gynecol Scand 2003;82: 867-70.

4. Gallegos F, Pavéz C, Jara M, Jesam C, Montero JC, Bustos JC. Embarazo ectópico intersticial complicado: urgencia ginecológica. Rev Chil Obstet Ginecol 2005;70:414-7.

5. Moawad NS, Mahajan ST, Moniz MH, et al. Current diagnosis and treatment of interstitial pregnancy. Am J Obstet Gynecol 2010;202:15-29.

6. Van Den Eeden SK, Shan J, Bruce C, Glasser M. Ectopic pregnancy rate and treatment utilization in a large managed care organization. Obstet Gynecol 2005;105:1052-7.

7. Poon LC, Emmanuel E, Ross JA, Johns J. How feasible is expectant management of interstitial ectopic pregnancy? Ultrasound Obstet Gynecol. 2014;43:31721.

8. Practice Committee of the American Society for Reproductive Medicine. Medical treatment of ectopic pregnancy. Fertil Steril 2006; 86:S96.
9. Medical management of ectopic pregnancy. ACOG Practice Bulletin 94. American College of Obstericians and Gynecologists, 2008.

10. Soriano D, Vicus D, Mashiach R, et al. Laparoscopic treatment of cornual pregnancy: a series of 20 consecutive cases. Fertil Steril 2008; 90: 839-43.

11. Zarhi J, Campaña C, Brito R, Stuardo P, Schalper J. Manejo laparoscópico conservador del embarazo ectópico cornual. Rev Chil Obstet Ginecol 2003;68:3641.

12. Takeda A, Koyama K, Imoto S, Mori M, Sakai K, Nakamura $\mathrm{H}$. Successful management of interstitial pregnancy with fetal cardiac activity by laparoscopicassisted cornual resection with preoperative transcatheter uterine artery embolization. Arch Gynecol Obstet 2009;280:305-8.

13. Murphy AA, Nager CW, Wujek KK, et al. Operative lapasocopy versus laparotomy for the management of ectopic pregnancy: a prospective trial. Fertil Steril 1992;57: 1180-5.

14. Larrain D, Marengo F, Bourdel N, et al. Proximal ectopic pregnancy: a descriptive general population-based study and results of different management options in 86 cases. Fertil Steril 2011; 95: 867-71.

15. Luengo-Tabernero A, Zornoza-García V, Luengo-Harto S, Lajas-Susañó JA. Embarazo ectópico cornual. Tratamiento eficaz con dosis única de metotrexato. Clin Invest Ginecol Obstet 2009;36:190-2. 